\title{
LITOS - a new balloon-borne instrument for fine-scale turbulence soundings in the stratosphere
}

\author{
A. Theuerkauf, M. Gerding, and F.-J. Lübken \\ Leibniz-Institute of Atmospheric Physics at the Rostock University, Kühlungsborn, Germany \\ Received: 11 July 2010 - Published in Atmos. Meas. Tech. Discuss.: 18 August 2010 \\ Revised: 16 December 2010 - Accepted: 26 December 2010 - Published: 20 January 2011
}

\begin{abstract}
We have developed a new compact balloon payload called LITOS (Leibniz-Institute Turbulence Observations in the Stratosphere) for high resolution wind turbulence soundings in the stratosphere up to $35 \mathrm{~km}$ altitude. The wind measurements are performed using a constant temperature anemometer (CTA) with a vertical resolution of $\sim 2.5 \mathrm{~mm}$, i.e. $2 \mathrm{kHz}$ sampling rate at $5 \mathrm{~m} / \mathrm{s}$ ascent speed. Thereby, for the first time, it is possible to study the entire turbulence spectrum down to the viscous subrange in the stratosphere. Including telemetry, housekeeping, batteries and recovery unit, the payload weighs less than $5 \mathrm{~kg}$ and can be launched from any radiosonde station. Since autumn 2007, LITOS has been successfully launched several times from the LeibnizInstitute of Atmospheric Physics (IAP) in Kühlungsborn, Germany $\left(54^{\circ} \mathrm{N}, 12^{\circ} \mathrm{E}\right)$. Two additional soundings were carried out in 2008 and 2009 in Kiruna, Sweden $\left(67^{\circ} \mathrm{N}, 21^{\circ} \mathrm{E}\right)$ as part of the BEXUS program (Balloon-borne EXperiments for University Students). We describe here the basic principle of CTA measurements and prove the validity of this method in the stratosphere. A first case study allows a clear distinction between non-turbulent regions and a turbulent layer with a thickness of some tens of meters. Since our measurements cover the transition between the inertial and viscous subrange, energy dissipation rates can be calculated with high reliability.
\end{abstract}

\section{Introduction}

Gravity waves and turbulence play a crucial role in the understanding of the energy budget, momentum transfer and trace gas distribution in the atmosphere. Breaking gravity waves produce turbulent structures in temperature and wind fields

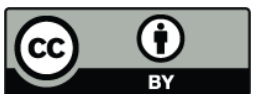

Correspondence to: A. Theuerkauf (theuerkauf@iap-kborn.de) and generate new gravity waves at the same time. Typically the stratosphere is statically stable and well stratified, due to its small negative or even positive temperature gradient. Nevertheless gravity waves can induce instability leading to wave breaking and turbulence.

Stratospheric turbulence is assumed to be weak on average compared to, for instance, mesospheric turbulence (e.g., Lübken, 1992; Hocking, 1990). Breaking gravity waves in the stratosphere however potentially modify the energy transfer from the troposphere into the mesosphere. Observations show that the vertical growth rate of gravity waves in the stratosphere is smaller than expected for undisturbed propagation (e.g., Rauthe et al., 2008). This implies that some of the wave energy is deposited in the stratosphere. Therefore this energy cannot contribute to the mesospheric energy budget. Moreover stratospheric turbulence is a potentially important process in the vertical mixing of trace species (e.g., Lilly et al., 1974).

Turbulence in the stratosphere appears on scales between some millimeters and several meters. Previous observations have shown that it occurs in thin isolated layers extending some ten or hundred meters in the vertical and some hundred kilometers in the horizontal (e.g., Barat, 1982; Sato and Woodman, 1982). The turbulent regions are vertically divided by sharp boundaries with non-turbulent regions.

Figure 1 shows a schematic energy spectrum for turbulence in the stratosphere. The spectrum is separated into the characteristic subranges which are based on the dominating physical processes. The transition between the inertial subrange and the viscous subrange defines the inner scale $l_{0}$. Typical values for $l_{0}$ in the stratosphere are in the range of centimeters. At very small scales (viscous subrange), the energy is dissipated into heat by viscosity. The aim of our measurements is to obtain this energy dissipation rate $\varepsilon$ and thereby quantify the influence of turbulence on the stratosphere. 


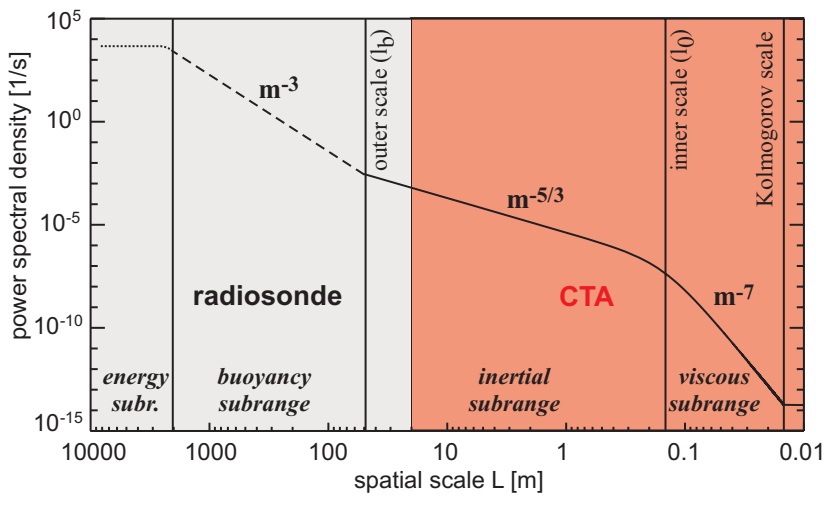

Fig. 1. Theoretical turbulent spectrum for $20 \mathrm{~km}$ altitude. In contrast to a standard radiosonde, the constant temperature anemometer (CTA) provides measurement of the spectrum down to the viscous subrange.

Remote sensing systems like radars, lidars and satellitebased sounders do not provide sufficient resolution to measure the turbulence spectrum down to the smallest scales or provide no signal at all in the middle stratosphere (e.g., Engler et al., 2005; Luce et al., 2002; Smalikho et al., 2005; Gurvich and Brekhovskikh, 2001; Sofieva et al., 2007). In-situ measurements are typically performed either below $15 \mathrm{~km}$ with aircraft and tethered lifting systems (e.g., Siebert et al., 2007; Frehlich et al., 2003; Balsley, 2008) or above $60 \mathrm{~km}$ with sounding rockets (e.g., Lübken et al., 2002). Thus in-situ high resolution balloon soundings still provide the only possibility for detailed observations of stratospheric turbulence. During the 1980s, pioneering work has been done by J. Barat and coworkers with balloon-borne ionic anemometers (e.g., Barat, 1982; Barat et al., 1984; Dalaudier et al., 1989). Their measurements resolved scales down to some ten centimeters (i.e. within the inertial subrange). But more precise turbulent energy dissipation rates can best be deduced by measurements covering the turbulent inner scale $l_{0}$ and part of the viscous subrange (Lübken et al., 1993). This implies that the spatial resolution must be at least $1 \mathrm{~cm}$ in the stratosphere. But those soundings are technically challenging and up to now stratospheric soundings quantifying turbulence are rare. In fact a sub-centimeter resolution has not been achieved yet.

We describe here an approach using a constant temperature anemometer (CTA) for high resolution balloon-borne wind soundings. The CTA allows us to measure wind fluctuations with a vertical resolution of $2.5 \mathrm{~mm}$. Therewith for the first time, a study of the whole turbulence spectrum down to the viscous subrange in the stratosphere is presented. Within this paper we concentrate on the presentation of a new technique for the estimation of energy dissipation rates in the stratosphere by use of CTA, while the geophysical interpretation will be the task of a subsequent paper. In the following section, we present the instrument design of LITOS and the measurement principle of the CTA sensor. Since CTA systems have never been used before in the stratosphere, we performed laboratory tests to investigate the dynamic characteristics of the sensor. The test procedures and the results are described in Sect. 3. The payload has been launched successfully several times from our site at Kühlungsborn (Germany, $54^{\circ} \mathrm{N}, 12^{\circ} \mathrm{E}$ ) and as part of the BEXUS 6 and BEXUS 8 payloads at Kiruna $\left(\right.$ Sweden, $67^{\circ} \mathrm{N}, 21^{\circ} \mathrm{E}$ ). First results of the BEXUS 6 flight (8 October 2008) including energy dissipation rates are presented in Sect. 4. Finally we discuss our results in Sect. 5.

\section{Experimental method}

As mentioned above the precise calculation of energy dissipation rates requires to resolve the inner scale, i.e. spatial scales of a few $\mathrm{cm}$. This measurement resolution can only be achieved by in-situ soundings. We developed the new balloon payload LITOS (Leibniz-Institute Turbulence Observations in the Stratosphere) which consists of a commercial CTA system plus a specially designed A/D converter, telemetry, tracking hardware and housekeeping electronics. Additionally a standard radiosonde (Vaisala RS92) provides atmospheric background profiles of wind, temperature, humidity and pressure.

The measurement principle of CTA is based on convective cooling caused by the air flow passing a heated thin wire (see Sect. 2.2). For our measurements we use a platinum plated tungsten wire (Type 55P03, Dantec Dynamics), which is $5 \mu \mathrm{m}$ in diameter and $1.25 \mathrm{~mm}$ long. The CTA signal output is A/D converted using a 16 bit ADC with 2000 samples per second resulting in a vertical resolution of $2.5 \mathrm{~mm}$ at $5 \mathrm{~m} / \mathrm{s}$ ascent speed. In an improved version of LITOS the sampling rate has been increased to $8000 \mathrm{~Hz}$, resulting in a $\sim 0.6 \mathrm{~mm}$ spatial resolution. (It should be noted here that the CTA system is generally specified up to $100 \mathrm{kHz}$.) The wire axis has been mounted vertically to achieve largest sensitivity for horizontal flow and less sensitivity for vertical flow due to the ascent of the balloon.

LITOS has been launched from Kühlungsborn with a special designed gondola of $35 \mathrm{~cm}$ side length. To minimize influences of the gondola on our measurements, the CTA sensor is placed $\sim 20 \mathrm{~cm}$ above the top of the payload (outside the shear layer around the box). Additionally the payload may be affected by pendulum and rotational motions. While the pendulum motions have comparatively long periods of $15 \mathrm{~s}$, the rotations may occur on different scales. In order to minimize the rotations we have attached a wind vane of about $40 \mathrm{~cm}$ diameter on a $70 \mathrm{~cm}$ boom to the payload box.

For the launches from Kiruna, LITOS has been integrated into the BEXUS gondola which has a side length of $60 \mathrm{~cm}$. In order to minimize influences of the gondola, we used a long tube $(2 \mathrm{~m}$ long) attached to one edge of the gondola. The CTA sensor was attached to the top of this tube and therefore 
was located $1.4 \mathrm{~m}$ above the gondola. Thus the influence of the gondola was minimized. As the BEXUS gondola is not actively stabilized, rotational and pendulum motions may influence the measurements. We studied the acceleration of the gondola measured by another instrument on the same gondola (BEXUS 6). Their measurement reveal a typical pendulum velocity of $1.5 \mathrm{~m} / \mathrm{s}$ (maximum $2 \mathrm{~m} / \mathrm{s}$ ) which affects the observation as a bias varying sinusoidal with a $20 \mathrm{~s}$ period. This bias is removed before further analysis. For further examinations of this possible bias a special developed housekeeping device measuring the rotation and pendulum motion of the gondola has been integrated in the latest version of the payload.

LITOS can be used as one-way instrument if the telemetry conditions are sufficient (no obstruction by trees, houses or terrain). If safe recovery of the instrument is assured, the payload can also be used with on-board data storage. More details about the LITOS payload can be found in Gerding et al. (2009).

In the next section we explain the general principle of turbulence measurements with balloons. Section 2.2 includes the description of constant temperature anemometry with a special focus on our application.

\subsection{Balloon measurement principle}

Due to its large diameter, the balloon follows the ambient wind field during the ascent phase (see Fig. 2). Ignoring pendulum motions for a moment, the payload at altitude $z$ is also following the wind field at balloon height at $z+h$. For a wind constant with altitude, the effective flow observed at payload height would be zero. Consequently, any variation of the wind as observed by our sensor is the difference between the wind vectors at balloon height $\vec{v}(z+h)$ and at payload height $\vec{v}(z)$. As a result, we obtain an altitude profile of the wind differences $\Delta \vec{v}$. In the following, we will use the term "wind" for the measured quantity instead of "wind difference" or "effective flow". Nevertheless it should be kept in mind that the absolute wind profile is only provided by the simultaneous radiosonde sounding ( $1 \mathrm{~s}$ resolution).

Additionally, we want to point out that we also considered the balloon wake effect described by e.g., Barat et al. (1984). As outlined there, the gondola will be outside the wake, if the distance between the gondola and the center of wake is $\geq 4$ times the radius $r$ of the balloon. The balloon in Kiruna has a volume of $10000 \mathrm{~m}^{3}$, i.e. a radius of $\sim 20 \mathrm{~m}$ (assuming a spherical shape) and the distance between the gondola and the balloon has been $50 \mathrm{~m}$. From the vertical shear of horizontal winds we calculated a distance between the gondola and the wake of at least $80 \mathrm{~m}$ and typically $>150 \mathrm{~m}$ during the ascent phase (except the first $3 \mathrm{~km}$ after launch). Thus we conclude that our measurements are not influenced by the balloon wake.

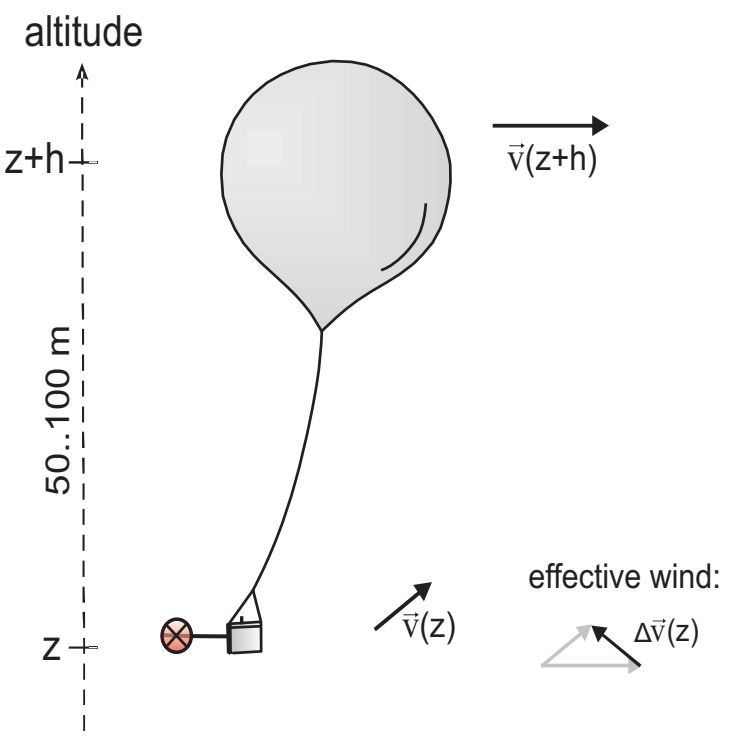

Fig. 2. Schematic drawing of the principle of balloon-borne wind turbulence soundings.

\subsection{Constant temperature anemometry}

Constant temperature anemometry is a well known and widely used technique for flow measurements in gases and liquids in laboratory. Their small size and light weight make CTA sensors particularly suitable for balloon-borne measurements. But so far they have never been used for stratospheric soundings. Regarding the general sensor behaviour, several extensive studies were performed and have given theoretical and semi-empirical descriptions of the measured signal (e.g., Bruun, 1970; Bruun et al., 1988; van Dijk and Nieuwstadt, 2004). We refer to the literature for more details and only emphasize the main aspects with a special focus on our application.

CTA measurements are based on the convective cooling of a heated thin wire (here: $5 \mu \mathrm{m}$ diameter, $1.25 \mathrm{~mm}$ length) caused by air flow passing the wire. The wire forms one leg of a Wheatstone bridge and is heated to a temperature of $\sim 500 \mathrm{~K}$. The Wheatstone bridge is balanced by controlling the current through the wire so that the resistance - and hence temperature - is kept constant. Changes in the ambient flow velocity cause changes in the convective heat loss of the wire and consequently, change the wire temperature, i.e. its resistance. The resulting difference in the bridge voltage therefore directly depends on the ambient flow velocity. Generally, the heat from the wire is transferred to the surrounding fluid by radiation, free convection, heat flow through the prongs and forced convection. The sum of these heat fluxes equals the supplied heat $\dot{Q}_{E}$, which is given by:

$$
\dot{Q}_{E}=I^{2} R=\frac{U^{2}}{R}=\dot{Q}_{\text {rad }}+\dot{Q}_{\text {freeconv }}+\dot{Q}_{\text {prongs }}+\dot{Q}_{\text {forcedconv }}
$$


where $U$ is the bridge voltage, $R$ the wire resistance and $I$ the electric current. Based on this relation, the further determination of the heat loss implies the definition of the heat transfer coefficient $\alpha$. The latter is expressed by the Nusselt number $N u$, which is a dimensionless number describing the convective heat transfer from a surface. In Appendix A, we discuss the determination of the heat loss of the wire and the difficulty in obtaining the correct definition of $N u$. Due to the complexity of the Nusselt number and the uncertainties in its determination, we would prefer the individual calibration of each wire. Later in this section we will describe that for the determination of turbulence parameters the wire calibration is not necessary. Nevertheless we shortly describe here the calibration principle as it provides important insights into CTA measurements.

During the calibration procedure, the wire is placed in a wind tunnel to adjust to different laminar flow velocities. As a result, a static calibration curve of the output voltages $U$ as a function of the flow velocities $v$ is obtained. Based on King (1914), the calibration data can be fitted by the modified King's law:

$U^{2}=A+B v^{n}$.

$A$ and $B$ are empirical calibration constants for each fluid. The exponent $n$ depends slightly on the flow velocity. According to Jørgensen (2002), $n=0.45$ is a recommended starting value. By determining these calibration constants, it is possible to convert the measured voltages to wind velocities.

The crucial point is that the calibration coefficients are only valid if the ambient conditions do not differ from those during the calibration. This concerns not only the wind conditions, but also the density, temperature and humidity (Cimbala and Park, 1990; Cardell, 1993; Durst et al., 1996; Hugo et al., 1999). Thus the calibration should be performed under ambient conditions similar to the conditions during the measurements. In our case, i.e. for stratospheric conditions, the density varies between $1.2 \mathrm{~kg} / \mathrm{m}^{3}$ and $\sim 1.0 \times 10^{-2} \mathrm{~kg} / \mathrm{m}^{3}$, the (relative) wind velocities are up to $2 \mathrm{~m} / \mathrm{s}$ and the temperature decreases to $\sim 200 \mathrm{~K}$. The water vapour mixing ratio in the stratosphere amounts to $\sim 5 \mathrm{ppm}$ and therefore the influence on our measurements is negligible (Durst et al., 1996).

We should point out here that for the analysis of turbulence, it is not necessary to derive absolute wind velocity values from the CTA signal. Instead, we perform a spectral analysis of the unscaled voltage signal to retrieve the spectral slope of the observed variations. Typically we analyse 8-20 s (i.e. $40-100 \mathrm{~m}$ ) of data for a single spectrum. Within this period, values like temperature, are sufficiently constant. From the spectral slope of the observed fluctuations, we deduce information about the stratospheric turbulence. For instance by determining the inner scale, we derive the energy dissipation rate $\varepsilon$. It is important to note, that $\varepsilon$ does not depend on absolute velocity values, but rather on the particular inner scale of the spectrum of relative fluctuations. However, no investigations for these wide pressure and temperature ranges have been done and the properties of the CTA sensor are completely unknown for stratospheric conditions. Therefore we performed laboratory tests within a climate and a vacuum chamber to check the response of the CTA on varying ambient conditions, i.e. temperature and pressure.

\section{Laboratory experiments of CTA response}

During all laboratory test procedures, we used a small wind calibration unit for CTA sensors, which we placed into a climate and a vacuum chamber. The wind calibration unit uses the effect of laminar flow behind a nozzle. By measuring the pressure drop across the nozzle the flow velocity can be calculated. Possible uncertainties of the calculated flow velocity can be caused by temperature changes during the experiment and by the measurement accuracy of the pressure sensors. The temperature has been observed during the experiments and its effect is considered in the equation for the determination of the flow velocity. Thus the remaining uncertainty is only caused by the accuracy of the pressure sensors, which is specified with $\pm 1 \%$. Hence, the velocity behind the nozzle is determined with an accuracy of $\pm 0.2 \mathrm{~m} / \mathrm{s}$. While knowing the exact flow velocities, we were able to set different values for temperature or pressure (i.e. density) of the flow passing the wire. Overall, we took measurements for a velocity range from 3 to $35 \mathrm{~m} / \mathrm{s}$ at pressure levels between 50 and $1000 \mathrm{hPa}$ and for a temperature range of 233 to $293 \mathrm{~K}$. Due to technical limitations of the wind calibration unit, we could not perform measurements below $3 \mathrm{~m} / \mathrm{s}$ and below $50 \mathrm{hPa}$.

\subsection{Temperature dependence of the CTA response}

Basically the heat transfer from the wire to the surrounding fluid is proportional to the temperature difference between the sensor and the fluid (see Appendix A, Eq. A1). From other experiments, it is known that the CTA response is influenced by temperature variations during the experiment (e.g., van Dijk and Nieuwstadt, 2004). For the correction of this temperature influence, different methods are suggested in the literature (e.g., Bruun, 1995; Jørgensen, 2002). But all correction methods are specified for only small temperature drifts and temperature ranges not found in the stratosphere. In other words, the CTA response has never been investigated below $\sim 280 \mathrm{~K}$. Therefore, we performed measurements within a climate chamber for the temperature range expected during a balloon flight, i.e. 233-293 K. Figure 3 (top) shows the voltage signal for various velocities obtained at different temperatures. The thin lines represent the King's law fits (according to Eq. 2) for each temperature level. As expected, the results reveal an influence of the temperature on the CTA response. For our examined temperature range, we found a maximum slope of $5 \mathrm{mV} / \mathrm{K}$, i.e. $0.23 \% / \mathrm{K}$. The question is now, whether this temperature influence will have an impact on the sensitivity of the wire response. In Fig. 3 (bottom) 

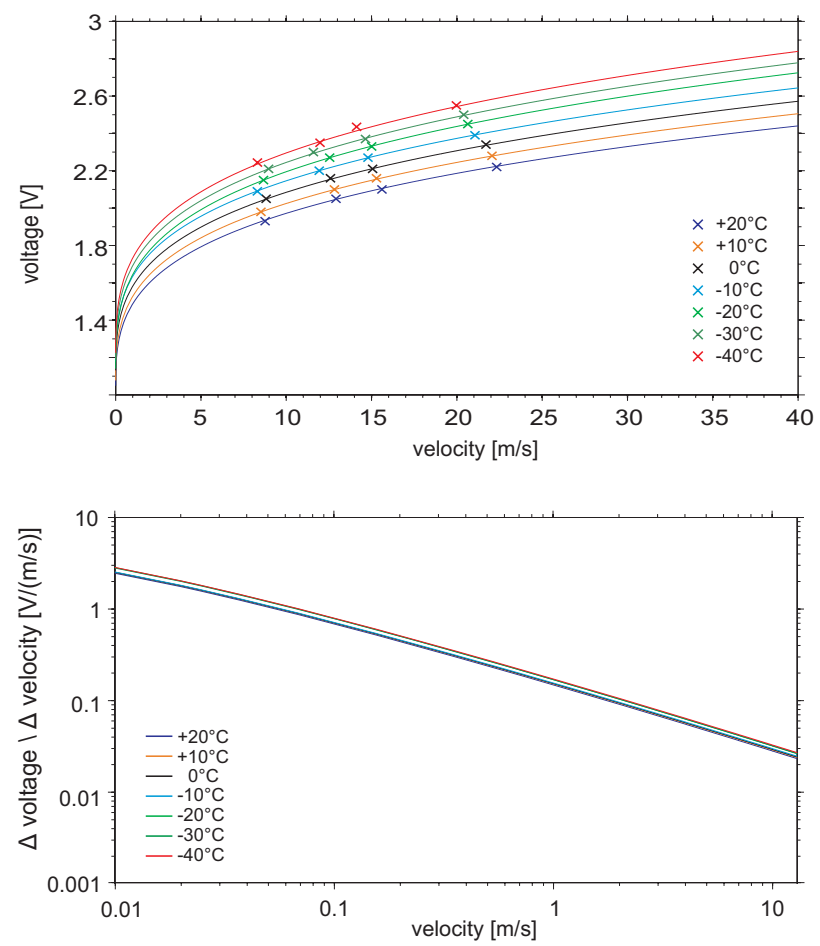

Fig. 3. Results of the climate chamber tests. The King's law (thin line) was fitted to the CTA voltage signals (data points) as a function of velocity for different temperatures (top). The bottom figure shows the sensitivity of the CTA signal as a function of velocity at different temperatures.

we therefore show the sensitivity ( $\Delta$ voltage/ $\Delta$ velocity) as a function of velocity for the different temperatures. Obviously the temperature has no significant effect on the sensor response. But, besides these findings, we noticed that the velocity considerably influences the sensor sensitivity. We will discuss this subject further in Sect. 5. Nevertheless, we have noted earlier that we use the unscaled voltage signal for the determination of the spectral slope of the turbulent fluctuations. Since the temperature does not affect the sensitivity of the sensor response, we do not have to correct the temperature influence. But if one would like to obtain absolute velocity fluctuations, the temperature influence has to be corrected before converting the voltage values to wind velocities. For the temperature correction the method given by e.g., Jørgensen (2002) can be used (after adjusting the exponent mentioned therein).

\subsection{Pressure dependence of the CTA response}

So far the pressure influence on the CTA response has been barely investigated and the few approaches do not cover pressure ranges we expect for stratospheric soundings (e.g., Hugo et al., 1999). In order to study the pressure influence on the sensor response, we therefore performed tests within a vacuum chamber. The temperature during the measurements
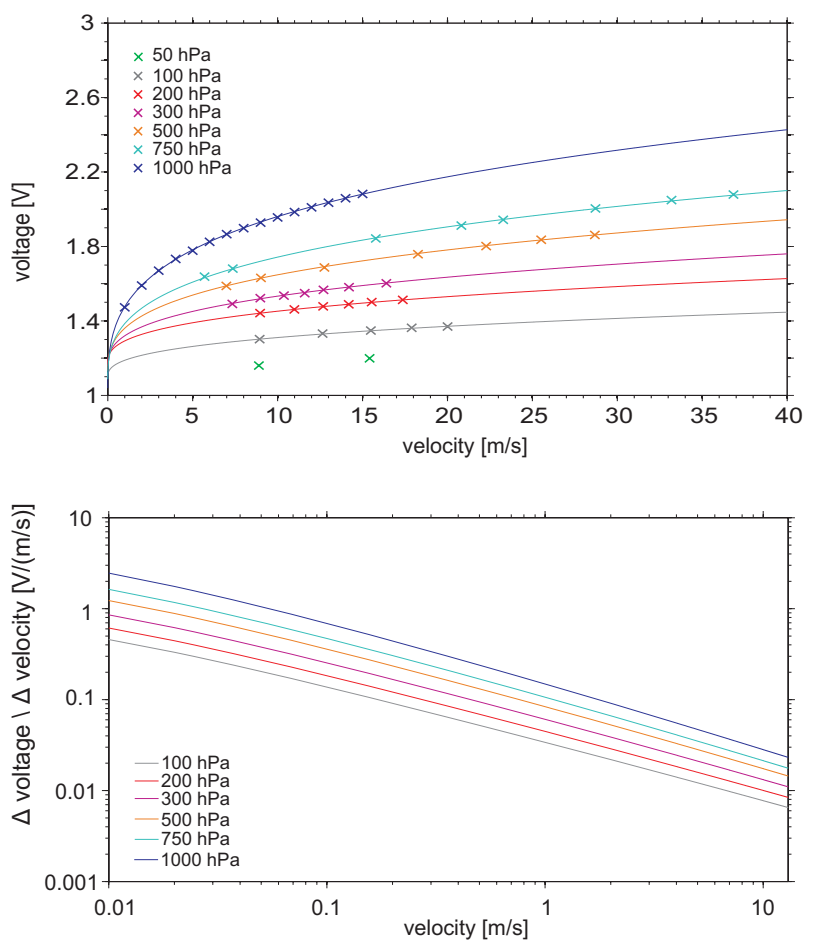

Fig. 4. Results of the vacuum chamber tests. The King's law (thin line) was fitted to the CTA voltage signals (data points) as a function of velocity for different pressure levels (top). At $50 \mathrm{hPa}$, only two points are obtained. Thus the King's law fit is omitted due to remaining ambiguities. The sensitivity of the CTA signal as a function of velocity at different pressure levels is shown in the bottom figure.

changed less than $5 \mathrm{~K}$ and therefore does not affect the results. In Fig. 4 (top) we present the voltage signal for various velocities together with the King's law fits (according to Eq. 2) represented by thin lines at different pressure levels. A direct pressure influence can easily be seen, as the slope of the King's law fit at $1000 \mathrm{hPa}$ varies significantly from the $100 \mathrm{hPa}$ curve. In the same way as for the temperature variations, we also show the sensitivity $\Delta$ voltage/ $\Delta$ velocity as a function of velocity for the different pressure levels. As can be seen from Fig. 4 (bottom) the sensitivity decreases with decreasing pressure and this effect is most evident at lower velocities. Again, the velocity directly influences the sensitivity of the sensor response and this effect is even much more pronounced than the pressure influence. However, using the results of the vacuum chamber tests we will demonstrate later in this paper (see Sect. 5) that the dependence of the sensitivity on the velocity has no significant impact on the spectral slopes of the turbulence measurements. Additionally, we show in the Appendix B that there exists an upper limit for the use of CTA sensors in the atmosphere around $\sim 1 \mathrm{hPa}(\sim 45 \mathrm{~km})$. During our measurements, however, we are sufficiently below this limitation. 
Table 1. Overview of launched LITOS payloads.

\begin{tabular}{lll}
\hline launch site & $\begin{array}{l}\text { Kühlungsborn } \\
\left(54^{\circ} \mathrm{N}, 12^{\circ} \mathrm{E}\right)\end{array}$ & $\begin{array}{l}\text { Kiruna } \\
\left(67^{\circ} \mathrm{N}, 21^{\circ} \mathrm{E}\right)\end{array}$ \\
\hline balloon weight/volume & $1200-3000 \mathrm{~g}$ & $10000 \mathrm{~m}^{3} / 12000 \mathrm{~m}^{3}$ \\
balloon type & rubber & $\begin{array}{l}\text { plastic } \\
\text { gondola weight }\end{array}$ \\
$\begin{array}{l}5 \mathrm{~kg} \\
\text { distance balloon-gondola }\end{array}$ & $100 \mathrm{~m}$ & $60 \mathrm{~kg} / 140 \mathrm{~kg}$ \\
max. altitude & $35 \mathrm{~km}$ & $29 \mathrm{~km}$ \\
\hline
\end{tabular}

\section{Balloon observations of turbulence}

We have successfully launched our payload LITOS six times since December 2007. Four soundings have been carried out from our site at the Leibniz-Institute of Atmospheric Physics (IAP) in Kühlungsborn (Germany, $54^{\circ} \mathrm{N}, 12^{\circ} \mathrm{E}$ ). In addition, two soundings took place at the Esrange Space Center in Kiruna (Sweden, $67^{\circ} \mathrm{N}, 21^{\circ} \mathrm{E}$ ) as part of the BEXUS (Balloon EXperients for University Students) campaigns in 2008 and 2009. The main differences between the launches from Kühlungsborn and Kiruna are given in Table 1.

For the launches from Kühlungsborn, we used rubber balloons whereas the launches from Kiruna were performed with plastic balloons. The different type and size of the balloon lead to different motions in the vertical and horizontal planes. For further investigation of this subject, we have developed a housekeeping device, including a 6-axis sensor, which measures the rotation and acceleration of the payload at a rate of $50 \mathrm{~Hz}$. This device has been part of recent launches at Kühlungsborn. Moreover, the disturbing effects of rotation and acceleration are also considered in the data analysis (see next section).

\subsection{Timeseries of signal fluctuations}

We now show some initial results of LITOS measurements obtained during the BEXUS 6 flight from Kiruna in 2008. As mentioned in Sect. 2 arbitrary movements of the gondola produce sinusoidal signal variations on temporal scales of some ten seconds. We eliminate these low frequency effects by removing a spline trend from the signal. During this launch, we observed many layers of turbulence. The vertical extent of these layers ranges from a few meters to some hundred meters, but normally do not exceed $500 \mathrm{~m}$. Figure 5 shows a typical example of such a turbulent layer obtained during the BEXUS 6 launch. The measured voltage fluctuations have been plotted as a function of altitude. It can easily be seen that at $\sim 26500 \mathrm{~m}$ the laminar flow suddenly became turbulent for $300 \mathrm{~m}$. The observation of those sharp boundaries between turbulent layers and non-turbulent regions is typical for the layered structure of stratospheric turbulence. Our findings are consistent with earlier observations of e.g., Sato and Woodman (1982) and Barat (1982). The small remaining fluctuations in the non-turbulent region are due to

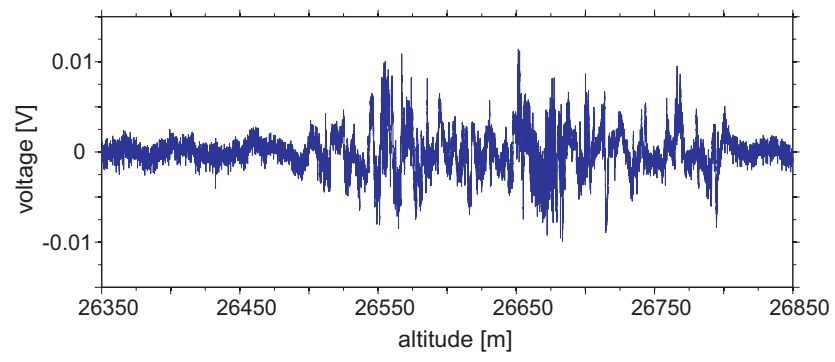

Fig. 5. Time record (converted to altitude) of the measured voltage fluctuations during the BEXUS 6 flight on 8 October 2008. Between 26500 and 26800 the gondola passes a turbulent layer, which is defined by sharp boundaries to the non-turbulent region.

instrumental noise and do not exceed $\sim 0.002 \mathrm{~V}$. These results demonstrate that our instrument works properly and is capable of detecting small-scale turbulent fluctuations.

\subsection{Spectral analysis of the BEXUS 6 timeseries}

As explained before, we obtain information about turbulence and turbulent energy dissipation rates from the spectral analysis of the measured relative voltage fluctuations. After removing a spline trend from the signal, we use the Welch method to calculate power spectral density values (Welch, 1967). To reduce spectral leakage, we apply a Hanning window function to the data before fitting. As a typical example, Fig. 6 (top) presents the spectrum for the turbulent region between $26550 \mathrm{~m}$ and $26650 \mathrm{~m}$ (see Fig. 5). The spatial scale $L$ is derived from $L=2 \pi / k=v_{\mathrm{b}} / f$ ( $k=$ wavenumber, $f=$ frequency, $v_{\mathrm{b}}=$ balloon ascent velocity). An $\mathrm{m}^{-5 / 3}$ slope is well identified between spatial scales of $8 \mathrm{~m}$ and $0.3 \mathrm{~m}$ as well as the transition to an $\mathrm{m}^{-7}$ slope below $0.1 \mathrm{~m}$. The observed slopes nicely agree with the slopes expected from theory (see Fig. 1). The noise level of our instrument starts at a power spectral density value of $\sim 10^{-7} \mathrm{~V}^{2} / \mathrm{s}$. This demonstrates that our instrument has the required resolution and sensitivity to cover the inertial and part of the viscous subrange. To our knowledge, these are the first in-situ measurements of turbulent spectra down to the viscous subrange in the stratosphere.

For comparison, the spectrum of the non-turbulent region between $26350 \mathrm{~m}$ and $26450 \mathrm{~m}$ (see Fig. 5) is shown in Fig. 6 (bottom). In contrast to the turbulent spectrum, the slope does not follow the characteristic $\mathrm{m}^{-5 / 3}$ behaviour. The power spectral densities for scales smaller than $1 \mathrm{~m}$ are much lower than in the turbulent case and basically show instrumental noise. At very small spatial scales there are still some apparent irregularities (in both spectra) presumably due to electronic disturbances. But they do not hamper the spectral analysis. Since the BEXUS gondola is not stabilised, slow rotation occurs. That pendulum and rotation would cause distinct peaks in the spectrum at lower frequencies 

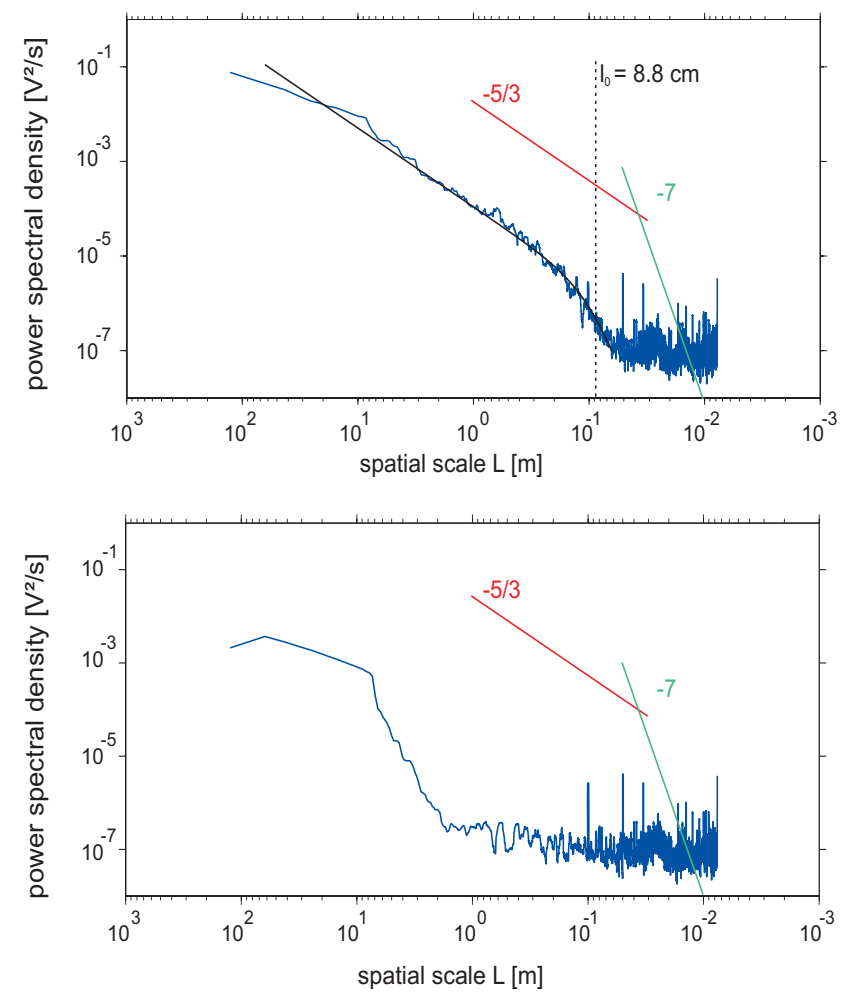

Fig. 6. Power spectrum of the voltage fluctuations for a turbulent region (26550-26 650 m) together with the best model fit (top). Values of $8.8 \mathrm{~cm}$ for the inner scale $l_{0}$ and $29 \mathrm{~mW} / \mathrm{kg}$ for the energy dissipation rate $\varepsilon$ are derived from the fit routine. The kinematic viscosity is $\nu=4.7 \times 10^{-4} \mathrm{~m}^{2} / \mathrm{s}$. The spectrum of a non-turbulent region (26350-26450 m) is shown for comparison (bottom).

( $\ll 1 \mathrm{~Hz})$. The measured spectra show no significant signal at that frequency.

\subsection{Calculation of energy dissipation rate}

From turbulent spectra, it is possible to derive relevant geophysical parameters like turbulent energy dissipation rates $\varepsilon$. Lübken et al. (1993) described a method to determine $\varepsilon$ from rocket-borne measurements of density fluctuations. Their routine includes the fitting of a theoretical spectrum model to the measured turbulent spectrum and from the best fit they obtain the energy dissipation rate $\varepsilon$. The theoretical model refers back to Heisenberg (1948) and exhibits an $\mathrm{m}^{-5 / 3}$ power law in the inertial subrange and an $\mathrm{m}^{-7}$ slope in the viscous subrange:

$W(\omega)=\frac{\Gamma(5 / 3) \sin (\pi / 3)}{2 \pi v_{b}} C_{v}^{2} \frac{\left(\omega / v_{b}\right)^{-5 / 3}}{\left\{1+\left(\left(\omega / v_{b}\right) / k_{0}\right)^{8 / 3}\right\}^{2}}$

where $\Gamma$ is the Gamma function $(\Gamma(5 / 3)=0.90167), v_{b}$ is the balloon velocity, $\omega=2 \pi f$ the cyclic frequency and $C_{v}^{2}$ the structure function. Based on this model it is possible to calculate the inner scale $l_{0}$, which characterises the transition between the inertial and the viscous subrange and basically determines the turbulent energy dissipation rate. Since the method presented by Lübken et al. (1993) is derived for density fluctuations and cannot directly be applied to velocity spectra, we accordingly adjusted certain equations (see Appendix C). As a result we obtain the following relation:

$l_{0}=11.35\left(\frac{v^{3}}{\varepsilon}\right)^{\frac{1}{4}}$

where $v$ is the kinematic viscosity. Taking into account that we analyse relative voltage fluctuations instead of absolute velocity fluctuations, we inserted a proportionality factor for the fitted equation. This factor basically scales the fitted spectrum upward or downward and has no influence on the determination of the energy dissipation rate, since $\varepsilon$ depends on the spatial scale of the break in the spectrum only. As an example we plotted the best fit model as a black line to the spectrum in Fig. 6 (top). The theoretical fit nicely agrees with our measured spectrum and we obtain an inner scale $l_{0}$ of $8.8 \mathrm{~cm}$ and an energy dissipation rate $\varepsilon$ of $29 \mathrm{~mW} / \mathrm{kg}$.

\section{Discussion}

The laboratory results shown in Sect. 3.2 reveal a non-linear dependence of the sensor sensitivity $\Delta$ voltage/ $\Delta$ velocity with respect to pressure and furthermore a decreasing sensitivity of the sensor response with decreasing pressure. In addition, the sensor sensitivity also varies for different wind velocities (Fig. 4 (bottom)). In other words, the sensor sensitivity depends not only on pressure but also on the relative background wind. Since we measure relative wind velocities up to $2 \mathrm{~m} / \mathrm{s}$, we are in the region with the largest influence of pressure and relative background wind (cf. Fig. 4 (bottom)). The question arises whether this could influence our turbulence measurements, i.e. the slope of the turbulence spectrum. Thus, we exemplarily normalized the voltage fluctuations between 26550 and $26650 \mathrm{~m}$ (see Fig. 5) to the relative background wind measured simultaneously by the radiosonde. Due to the lower sampling rate of the radiosonde data $(1 \mathrm{~s})$ we interpolated the values to the sampling rate of the measured voltage fluctuations $(0.5 \mathrm{~ms})$ and therewith derived a value of the relative background wind for each value of the fluctuations. Based on the $100 \mathrm{hPa}$ curve (Fig. 4 (bottom)) we then determined an individual correction factor for the sensor sensitivity of each value along the data sequence according to the relative wind at $26550 \mathrm{~m}$, i.e. the first value of the sequence. In fact, by using the $100 \mathrm{hPa}$ curve for this sequence, we overestimated the influence of the relative background wind. As can be seen from Fig. 4 (bottom), the sensitivity change decreases with decreasing pressure, so at an altitude of $26550 \mathrm{~m}$ (about $18 \mathrm{hPa}$ ) it is very likely that the sensitivity varies less than at $100 \mathrm{hPa}$. Unfortunately, due to technical limitation of the wind calibration unit, we could not 


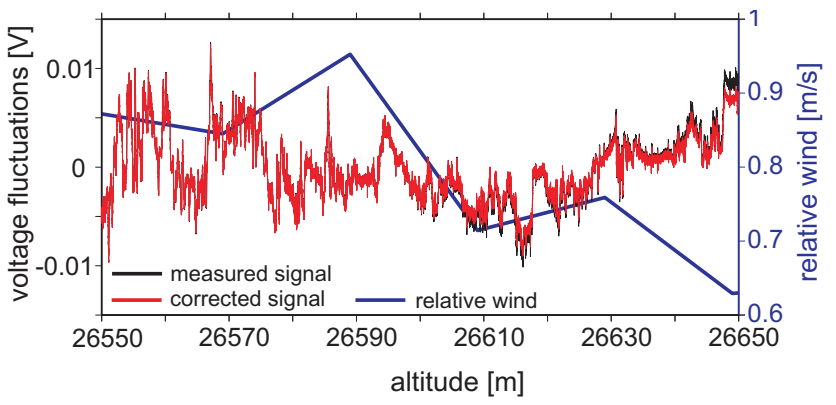

Fig. 7. Voltage fluctuations for the altitude region of $26550 \mathrm{~m}-$ $26650 \mathrm{~m}$ (see Fig. 5). The black line shows the measured fluctuations and the red line shows the fluctuation corrected for changes of background relative wind. The blue line presents the relative wind measured by radiosonde (used for the correction).

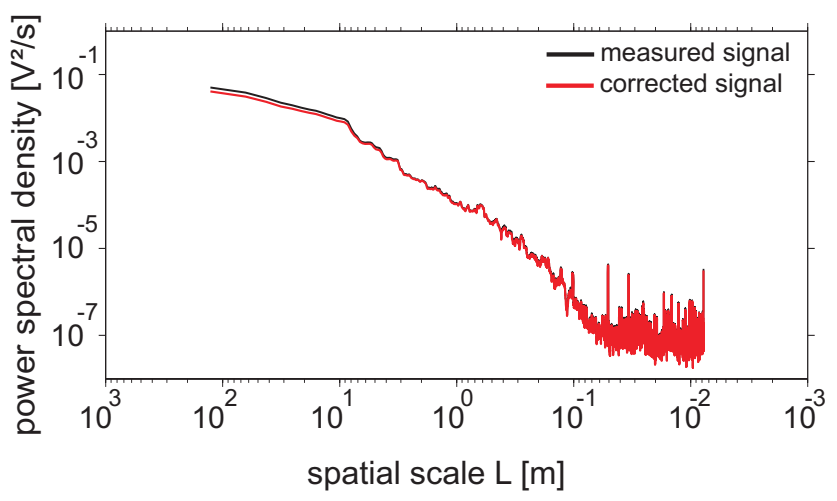

Fig. 8. Spectra of the corrected (red) and uncorrected (black) voltage fluctuations from Fig. 7.

obtain measurements of pressure values below $100 \mathrm{hPa}$. (The $50 \mathrm{hPa}$ values are omitted due to remaining ambiguities.)

Nevertheless, by applying the individual correction factor (obtained at $100 \mathrm{hPa}$ ) to the measured voltage fluctuations in the sequence, we eliminated the influence of the background relative wind. Figure 7 shows the uncorrected signal (black) and the corrected signal (red) together with the relative wind measured by the radiosonde (blue). The influence of the relative background wind is obviously rather small. Furthermore the spectra of the corrected and uncorrected signals are quite similar (Fig. 8). Here the largest deviations appear at larger spatial scales and decrease down to smaller scales. The resulting energy dissipation rates for the corrected and uncorrected spectra deviate by $\sim 2 \%$. Thus it is well justified to use the uncorrected signal for the determination of turbulence parameters.

Finally, we would like to discuss possible uncertainties within the determination of the energy dissipation rate. Small errors in the determination of the inner scale $l_{0}$ lead to significant uncertainties of $\varepsilon$, because $l_{0} \propto \varepsilon^{1 / 4}$. The conversion of frequency scales into spatial scales can be affected by the relative movement of the gondola. For that reason we included an accelerometer in our payload. However, for the conversion the particular ascent rate is used, which is modulated by the relative horizontal wind by the root of the sum of squared velocities (vertical and relative horizontal). For the BEXUS 6 launch we therefore estimated an error of the inner scale due to ignorance of relative horizontal wind of $\sim 10 \%$ resulting in $30-50 \%$ error in $\varepsilon$. This error can be significantly reduced by careful analysis of the acceleration data. A second error source is the fact, that the data sequence taken for a single turbulence spectrum is not necessarily filled with homogeneous turbulence. Typically spectra are calculated from $40-100 \mathrm{~m}$ sections of data. The turbulent layer has to fill a large part of this section to produce a clear spectral signal. Nevertheless inhomogeneous turbulence might result in some smearing of the spectra especially in the transition region of inertial and viscous subrange even for this high resolved observations. By this the determination of the inner scale might be affected and thus also the energy dissipation rate. For turbulent layers thinner than $40-100 \mathrm{~m}$ we assume a maximum error of $\sim 10 \%$ for the inner scale, similar to the factor induced by ignoring the relative horizontal wind. Nevertheless, we would like to point out that in contrast to other methods, the calculation of $\varepsilon$ does not depend on absolute spectral densities (which may be affected by changing instrument sensitivities) but only on the precise measurement of the temporal variations. The range of $\varepsilon$ values which can be observed with LITOS vary between $2.1 \times 10^{-6} \mathrm{~W} / \mathrm{kg}$ (maximum $l_{0}$ of $1 \mathrm{~m}$ ) and $5.3 \mathrm{~W} / \mathrm{kg}$ (minimum $l_{0}$ of $0.025 \mathrm{~m}$ ). We would like to underline that this range of energy dissipation rates can be measured by LITOS from the boundary layer up to the middle stratosphere, as the noise level is constant with altitude.

\section{Summary and conclusions}

In this paper, we presented the first results of a new compact balloon-borne instrument called LITOS (Leibniz-Institute Turbulence Observations in the Stratosphere). LITOS measures wind fluctuations in the stratosphere with very high vertical resolution $(2.5 \mathrm{~mm})$. The first launch took place on 12 December 2007 and the instrument has been improved further since. A constant temperature anemometer (CTA) is the main part of the balloon payload LITOS, which has a total weight of less than $5 \mathrm{~kg}$ and can be launched from any radiosonde station.

There is very little knowledge in the literature about CTA measurements at low pressures and we have performed our own laboratory studies within a vacuum chamber. The studies show a decreasing sensitivity with decreasing pressure. Nevertheless we have shown that for our purpose, the CTA technique is well suited. The main goal of our study is to obtain geophysical parameters like the turbulent energy dissipation rate. This rate only depends on the particular inner scale of the spectrum of relative fluctuations. Thus we can 
deduce information about the stratospheric turbulence from the unscaled voltage signal.

We have shown results of the BEXUS 6 flight launched from Kiruna on 8 October 2008, where we observed several turbulent layers with a vertical extent ranging from a few meters to some hundred meters. There are sharp boundaries between turbulent and quiet regions. Our observations confirm the findings of e.g., Barat (1982). The measurement resolution has been significantly improved compared to the soundings by Barat and coworkers in 1980s. Due to the highly improved resolution of our measurements, we are able to measure the turbulent spectrum in the stratosphere down to viscous subrange. To our knowledge this was not done before. The turbulent spectra of the measured voltage fluctuations nicely agree with the theoretically expected behaviour of turbulence in the inertial and viscous subrange. For the determination of the energy dissipation rate, we have adapted the spectral model method of (Lübken et al., 1993). Since this method was derived for density fluctuations we accordingly adjusted the theory. By fitting the spectral model to the spectrum of voltage fluctuations, we determine the inner scale and therewith the energy dissipation rate. For the presented example of a turbulence spectrum measured by LITOS, the fitting procedure yields an energy dissipation rate of $29 \mathrm{~mW} / \mathrm{kg}$ and an inner scale of $8.8 \mathrm{~cm}$. The value of $\varepsilon$ deviates by a factor of up to 100 from earlier indirect measurements. Further geophysical analysis like variation of epsilon, turbulent layer thickness and distances will be the task of a subsequent paper. Additionally, future launches of LITOS are planned to obtain a "climatology" of stratospheric turbulence and to characterise selected wave breaking situations.

\section{Appendix A}

\section{Theoretical determination of the heat transfer}

The heat transfer from the wire to the surrounding fluid is a sum of the heat flow due to forced convection, free convection, radiative cooling and heat flow through the prongs (see Eq. 1). Due to the small size of the heated wire, radiative cooling $\dot{Q}_{\text {rad }}$ is considerably smaller than the heat which is transferred from the sensor by e.g. forced convection $\dot{Q}_{\text {forcedconv }}$ (Durst, 2008). For the case of the BEXUS 6 launch the heat transfer due to radiation amounts to a maximum factor of $1.3 \%$ of the total heat transfer. Consequently, the heat loss resulting from radiation can be neglected.

According to Collis and Williams (1959), free convection $\dot{Q}_{\text {freeconv }}$ can also be omitted, if

$\operatorname{Re}>G r^{1 / 3}$

where $R e$ is the Reynolds number

$$
R e=\frac{v d_{\mathrm{w}}}{v}
$$

( $v=$ flow velocity, $d_{\mathrm{w}}=$ wire diameter and $\nu=$ kinematic viscosity of the fluid) and the Grashof number $\mathrm{Gr}$

$G r=g\left(T_{\mathrm{w}}-T_{\mathrm{a}}\right) \frac{d_{\mathrm{w}}^{3}}{v^{2} T_{\mathrm{a}}}$,

( $g$ is the gravitation acceleration, $T_{\mathrm{w}}$ the wire temperature and $T_{\mathrm{a}}$ the temperature of the ambient fluid). To verify Eq. (A1), we calculated the Grashof number and the Reynolds number using typical data from our soundings. Throughout the entire ascent phase, $R e$ is much larger than $\mathrm{Gr}^{1 / 3}$ and therefore we can omit the free convection term in the heat transfer Eq. (1). To minimize the (artificial) heat flow from the tungsten wire to the prongs $\left(\dot{Q}_{\text {prongs }}\right)$, the wire has gold-plated ends connecting it with the wire prongs. Nevertheless, for typical CTA applications, the heat loss to the prongs amounts to about $10-20 \%$ of the total heat loss from the sensor (Durst, 2008). Thus the heat flow through the prongs is considered to be proportional to the forced convective flow and Eq. (1) simplifies to

$\dot{Q}_{E} \approx c \cdot \dot{Q}_{\text {forcedconv }}$

where $c$ is a constant.

Finally according to Eq. (1) we get

$U^{2}=c R \dot{Q}_{\text {forcedconv }}$.

The measured voltage signal is thus directly related to the heat loss through forced convection. The latter is defined as:

$\dot{Q}_{\text {forcedconv }}=\alpha \pi l_{\mathrm{w}} d_{\mathrm{w}}\left(T_{\mathrm{w}}-T_{\mathrm{a}}\right)$,

where $\alpha$ is the heat-transfer coefficient and $l_{\mathrm{w}}$ the wire length. The heat-transfer coefficient is given by

$\alpha=\frac{N u k}{d_{\mathrm{w}}}$

where $N u$ is the Nusselt number and $k$ represents the heat conduction of the fluid. The Nusselt number is defined as

$N u=N u\left(R e, G r, K n, P r, M a, l_{\mathrm{w}} / d_{\mathrm{w}}, \delta T_{\mathrm{a}}, \ldots\right)$.

By inserting Eqs. (A4), (A6) and (A7) in Eq. (1) one obtains for the measured voltage

$U^{2}=c N u k \pi l_{\mathrm{w}} R\left(T_{\mathrm{w}}-T_{\mathrm{a}}\right)$.

For further examination, $N u$ has to be determined individually for every used wire and the flow properties described by e.g. $R e, G r$, the Knudsen number $K n$, the Prandtl number $P r$ and the Mach number $M a$. There exist several approaches in the literature for the empirical estimation of the Nusselt number for specific flow conditions (e.g., Collis and Williams, 1959; Cimbala and Park, 1990; Durst et al., 1996). The advantage of those formulations of $N u$ is that the heat loss from the wire and the dependence on the flow velocity can be obtained without calibration. However, a precise knowledge of all influencing parameters can not be provided with sufficient accuracy. For example, parameters of the wire (i.e. effective length and diameter) are not available with the required precision due to the complicated process of the wire manufacturing. 


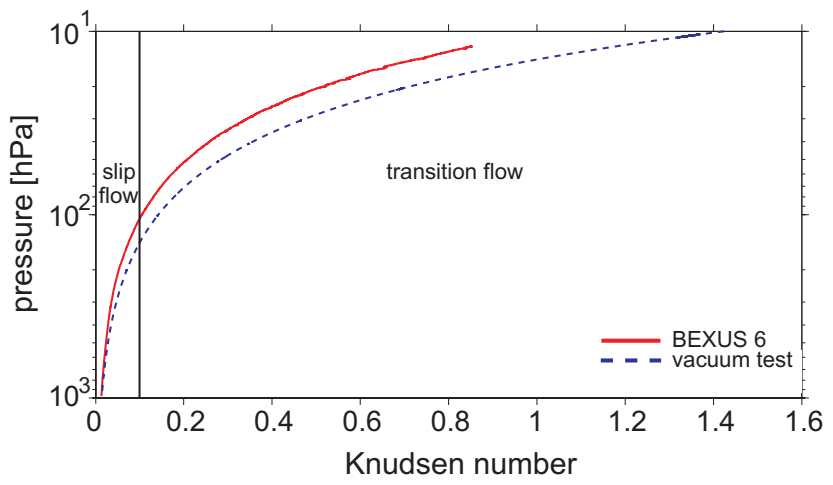

Fig. B1. Knudsen number for the vacuum test and for the BEXUS 6 flight.

\section{Appendix B}

\section{Knudsen number}

Figure 4 (bottom) raises the questions whether the sensor sensitivity decreases further at pressures below $100 \mathrm{hPa}$ and whether there is a lower pressure limit for CTA soundings.

Furthermore the heat-transfer equation for the interpretation of the CTA signal (as explained in Appendix A) requires continuum flow conditions. We therefore have to investigate the flow conditions during the balloon ascent phase. By determining the Knudsen number $K n$, it is possible to assess whether continuum flow approximations are applicable or not. The Knudsen number is defined as the dimensionless ratio of the mean free path $\lambda$ to a characteristic length scale, in our case the wire diameter $d_{\mathrm{w}}$,

$K n=\lambda / d_{\mathrm{w}}$.

The flow regime can be divided into the continuum flow $\left(K n<10^{-2}\right)$, the slip flow regime $\left(10^{-2}<K n<10^{-1}\right)$, the transition regime $\left(10^{-1}<K n<10\right)$ and the free molecular flow for $K n>10$ (e.g., Devienne, 1965). Figure B1 shows the Knudsen number for our vacuum tests and for the BEXUS 6 flight. Typically CTA measurements are performed in the slip flow regime, where continuum flow equations are still appropriate. With decreasing pressure, the heat transfer to the surrounding medium by convection decreases. Finally, in the free molecular flow, continuum approximations are no longer applicable. Therewith, a lower pressure limit for CTA soundings can be estimated when $K n>10$, i.e. at $\sim 1 \mathrm{hPa}$ $(\sim 45 \mathrm{~km})$. As can be seen from Fig. B1 in our case, the Knudsen number is well below 10, i.e. our measurements do not occur in the free molecular flow regime. Hence, for our purpose, CTA is well suitable since the measurement principle itself is valid.

\section{Appendix C}

\section{Derivation of the inner scale from the Heisenberg spectrum}

For the calculation of the energy dissipation rate we have adopted the method of Lübken (1992) and Lübken et al. (1993) which was formulated for density fluctuations. Since we measure velocity fluctuations and not density fluctuations we have to modify certain equations. Basically the theoretical spectrum is derived from the Heisenberg model. This model exhibits an $\mathrm{m}^{-5 / 3}$ power law in the inertial subrange and a smooth transition to the $\mathrm{m}^{-7}$ power law in the viscous subrange. The 1-dimensional frequency spectrum is given by:

$W(\omega)=\frac{\Gamma(5 / 3) \sin (\pi / 3)}{2 \pi v_{b}} C_{v}^{2} \frac{\left(\omega / v_{b}\right)^{-5 / 3}}{\left\{1+\left(\left(\omega / v_{b}\right) / k_{0}\right)^{8 / 3}\right\}^{2}}$

where $\Gamma$ is the Gamma function $(\Gamma(5 / 3)=0.90167), v_{b}$ is the balloon velocity and $\omega=2 \pi f$ is the cyclic frequency. $C_{v}^{2}$ is the structure function constant and for velocity fluctuations it is given by:

$C_{v}^{2}=4 \alpha \varepsilon^{2 / 3}$

(e.g. Barat and Bertin, 1984; Bertin et al., 1997; Hocking, 1999). The empirical constant $\alpha$ is taken as 0.5 (Bertin et al., 1997; Antonia et al., 1981). The Heisenberg model "breaks" at $k_{0}$, which is the intersection of the asymptotic form of $W(\omega)$ in the inertial and viscous subrange. Tatarskii (1971) has shown that $k_{0}$ is determined from the behaviour of the structure function $D$ at the origin and that the structure function is related to the 3-dimensional spectrum by:

$\frac{d^{2}}{d r^{2}} D(0)=\frac{8 \pi}{3} \int_{0}^{\infty} \Phi(k) k^{4} \mathrm{~d} k$

Since our measurement axis is perpendicular to the propagation direction, we use the transversal structure function for velocity fluctuations:

$D(r)=\frac{2}{15} \frac{\varepsilon}{v} r^{2}$

(Tatarskii, 1971). Thus Eq. C3 forms to:

$\frac{4}{15} \frac{\varepsilon}{v}=\frac{8 \pi}{3} \int_{0}^{\infty} \Phi(k) k^{4} \mathrm{~d} k$

Assuming an isotropic "frozen" random field, the 1dimensional spectrum can be converted to the 3-dimensional spectrum (Tatarskii, 1971; Lübken et al., 1993):

$\Phi(k)=-\frac{v_{b}^{2}}{2 \pi k} \cdot \frac{d}{d \omega} W(\omega)$

Inserting Eq. C1 and Eq. C2 in Eq. C6 gives:

$$
\Phi(k)=\frac{5 \Gamma(5 / 3) \sin (\pi / 3) \cdot \varepsilon^{2 / 3}}{6 \pi^{2}} \cdot k^{-\frac{11}{3}} \cdot \frac{1+\frac{21}{5}\left(k / k_{0}\right)^{\frac{8}{3}}}{\left\{1+\left(k / k_{0}\right)^{\frac{8}{3}}\right\}^{3}}
$$


Combining Eq. C7 and Eq. C5 and rearranging for $k_{0}$ finally leads to the inner scale $l_{0}=2 \pi / k_{0}$ :

$$
l_{0}=2 \pi \cdot\left(\frac{45 \Gamma(5 / 3) \sin (\pi / 3)}{8}\right)^{\frac{3}{4}}\left(\frac{v^{3}}{\varepsilon}\right)^{\frac{1}{4}}=11.35 \cdot\left(\frac{v^{3}}{\varepsilon}\right)^{\frac{1}{4}}
$$

where $\eta=\left(v^{3} / \varepsilon\right)^{1 / 4}$ is the Kolmogorov microscale.

Acknowledgements. We acknowledge the support by the Leibniz graduate school ILWAO, jointly funded by the MBWK (for the government of Mecklenburg-Vorpommern) and the BMBF (for the German federal government). Additionally, we thank SNSB (Swedish National Space Board) and DLR (German Aerospace Center) for the possibility to be part of the BEXUS campaign. We thank our colleague Torsten Köpnick (IAP) for his support in hardware development.

Edited by: J.-P. Pommereau

\section{References}

Antonia, R. A.: Chambers, A. J., and Satyaprakash, B. R.: Kolmogorov constants for structure functions in turbulent shear flows, Q. J. Roy. Meteorol. Soc., 107, 579-589, 1981.

Balsley, B. B.: The CIRES Tethered Lifting System: a survey of the system, past results and future capabilities, Acta Geophys., 56, 21-57, 2008.

Barat, J.: Some characteristics of clear air turbulence in the middle stratosphere, J. Atmos. Sci., 39, 2553-2564, 1982.

Barat, J., Cot, C., and Sidi, C.: On the measurement of the turbulence dissipation rate from rising balloons, J. Atmos. Oceanic Technol., 1, 270-275, 1984.

Barat, J. and Bertin, F.: Simultaneous measurements of temperature and velocity fluctuations within clear air turbulence layers: Analysis of the estimate of dissipation rate by remote sensing techniques, J. Atmosp. Sci., 41, 1613-1619, 1984.

Bertin, F., Barat, J., and Wilson, R.: Energy dissipation rates, eddy diffusivity, and the Prandtl number: An in situ experimental approach and its consequences in radar estimate of turbulent parameters, Radio Sci., 32, 791-804, 1997.

Bruun, H.: Hot-Wire Anemometry - Principles and Signal Analysis, Oxford Science Publication, Oxford, 1995.

Bruun, H. H.: Interpretation of a hot wire signal using a universal calibration law, J. Phys. E: Sci. Instrum., 4, 225-231, 1970.

Bruun, H. H., Khan, M. A., Al-Kayiem, H. H., and Fardad, A. A.: Velocity calibration relationships for hot-wire anemometry, J. Phys. E: Sci. Instrum., 21, 225-232, 1988.

Cardell, G.: A note on the temperature-dependent hot-wire calibration method of Cimbala and Park, Exp. Fluids, 14, 283-285, 1993.

Cimbala, J. M. and Park, W. J.: A direct hot-wire calibration technique to account for ambient temperature drift in incompressible flow, Exp. Fluids, 8, 299-300, 1990.

Collis, D. C. and Williams, J.: Two-dimensional convection from heated wires at low Reynolds numbers, J. Fluid Mech., 6, 357389,1959

Dalaudier, F., Crochet, M., and Sidi, C.: Direct comparison between in situ and radar measurements of temperature fluctuation spectra: a puzzling result, Radio Sci., 24, 311-324, 1989.
Devienne, F. M.: Low-density heat transfer, Adv. Heat Transfer, 2, 271-356, 1965.

Durst, F.: An Introduction to the Theory of Fluid Flows, SpringerVerlag, Berlin, Germany, 676-694, 2008.

Durst, F., Noppenberger, S., Sill, M., and Venzke, H.: Influence of humidity on hot-wire measurements, Meas. Sci. Technol., 7, 1517-1528, 1996.

Engler, N., Latteck, R., Strelnikov, B., Singer, W., and Rapp, M.: Turbulent energy dissipation rates observed by Doppler MST Radar and by rocket-borne instruments during the MIDAS/MaCWAVE campaign 2002, Ann. Geophys., 23, 1147 1156, doi:10.5194/angeo-23-1147-2005, 2005.

Frehlich, R., Meillier, Y., Jensen, M. L., and Balsley, B.: Turbulence measurements with the CIRES tethered lifting system during CASES-99: Calibration and spectral analysis of temperature and velocity, J. Atmos. Sci., 60, 2487-2495, 2003

Gerding, M., Theuerkauf, A., Suminska, O., Köpnick, T., and Lübken, F.-J.: Balloon-borne hot wire anemometer for stratospheric turbulence soundings, Proceedings of the 19th ESA Symposium on European Rocket and Balloon Programmes and Related Research, SP-671, 175-180, 2009.

Gurvich, A. S. and Brekhovskikh, V. L.: Study of the turbulence and inner waves in the stratosphere based on the observations of stellar scintillations from space: a model of scintillation spectra, Waves Random Complex, 11, 163-181, 2001.

Heisenberg, W.: Zur statistischen Theorie der Turbulenz, (in German), Z. Phys., 124, 628-657, 1948.

Hocking, W.: Turbulence in the region $80-120 \mathrm{~km}$, Adv. Space Res., 10(12), 153-161, 1990.

Hocking, W.: The dynamical parameters of turbulence theory as they apply to middle atmosphere studies, Earth Planets Space, 51, 525-541, 1999.

Hugo, R. J., Nowlin, S. R., Eaton, F. D., Bishop, K. P., and McCrae, K. A.: Hot-wire calibration in a non-isothermal incompressible pressure variant flow, Proceedings of the SPIE symposium "Airborne Laser Advanced Technlogy II”, 3706, 11-23, 1999.

Jørgensen, F.: How to measure turbulence with hot-wire anemometers a practical guide, Publication 9040U6151, Dantec Dynamics A/S, Skovlunde, Denmark, 2002.

King, J. V.: On the convection of heat from a small cylinder in a stream of fluid: determination of the convection constant of small platinum wires with application to hot-wire anemometry, Phil. Trans. R. Soc., 214, 373-432, 1914.

Lilly, D. E., Waco, D. E., and Adelfang, S. I.: Stratospheric mixing from high-altitude turbulence measurements, J. Appl. Meteor., 13, 488-493, 1974.

Lübken, F., Rapp, M., and Hoffmann, P.: Neutral air turbulence and temperatures in the vicinity of polar mesosphere summer echoes, J. Geophys. Res., 107, 4273-4277, 2002.

Lübken, F.-J.: On the extraction of turbulent parameters from atmospheric density fluctuations, J. Geophys. Res., 97, 20385-20395, 1992.

Lübken, F.-J., Hillert, W., Lehmacher, G., and von Zahn, U.: Experiments revealing small impact of turbulence on the energy budget of the mesosphere and lower thermosphere, J. Geophys. Res., 98, 20369-20384, 1993.

Luce, H., Fukao, S., Dalaudier, F., and Crochet, M.: Strong mixing events observed near the tropopause with the MU radar and high resolution balloon techniques, J. Atmos. Sci., 59, 2885-2896, 
2002

Rauthe, M., Gerding, M., and Lübken, F.-J.: Seasonal changes in gravity wave activity measured by lidars at mid-latitudes, Atmos. Chem. Phys., 8, 6775-6787, doi:10.5194/acp-8-6775-2008, 2008.

Sato, T. and Woodman, R.: Fine altitude resolution observations of stratospheric turbulent layers by the Arecibo $430 \mathrm{MHz}$ radar, J. Atmos. Sci., 39, 2546-2552, 1982.

Siebert, H., Lehmann, K., and Shaw, R.: On the use of hot-wire anemometers for turbulence measurements in clouds, J. Atmos. Ocean. Technol., 24, 980-993, 2007.

Smalikho, I., Kopp, F., and Rahm, S.: Measurement of atmospheric turbulence by $2-\mu$ Doppler lidar, J. Atmos. Ocean. Technol., 22, 1733-1747, 2005.
Sofieva, V. F., Gurvich, A. S., Dalaudier, F., and Kan, V.: Reconstruction of internal gravity wave and turbulence parameters in the stratosphere using GOMOS scintillation measurements, J. Geophys. Res., 112, D12113, doi:10.1029/2006JD007483, 2007.

Tatarskii, V. I.: The effects of the turbulent atmosphere on wave propagation, Israel Program for Scientific Translations, Jerusalem, 1971.

van Dijk, A. and Nieuwstadt, F. T. M.: The calibration of (multi-) hot - wire probes: 1. temperature calibration, Exp. Fluids, 36, 540-549, 2004.

Welch, P. D.: The use of fast fourier transform for the estimation of power spectra: A method based on time averaging over short, modified periodograms, IEEE T. Acoust. Speech, AU-15, 70-73, 1967. 